\title{
Substance use Treatment and Harm Reduction Services for Migrants and Refugees in the COVID-19 Pandemic: Findings from a Global Survey
}

\section{Cornelis De Jong}

Radboud University Nijmegen: Radboud Universiteit

Ali Farhoudian

Tehran University of Medical Sciences

Mehrnoosh Vahidi

Tehran University of Medical Sciences

Mohsen Ebrahimi

Materials and Energy Research Center (MERC)

Hamed Ekhtiari

Laureate Institute for Brain Research

Parnian Rafei ( $\sim$ parnianrafeirafei@gmail.com )

University of Tehran, Tehran, IR https://orcid.org/0000-0002-4770-4801

Anja Busse

United Nations Office on Drugs and Crime

\section{Kathleen Brady}

Medical University of South Carolina

Masud Yunesian

Tehran University of Medical Sciences

\section{Nadine Ezard}

University of New South Wales

\section{Ramin Radfar}

University of California Los Angeles

\section{Alexander Baldacchino}

University of St Andrews

\section{Short report}

Keywords: Addiction, Harm Reduction, Migrants, Refugees, COVID-19, Pandemic

Posted Date: December 10th, 2020

DOI: https://doi.org/10.21203/rs.3.rs-123740/v1

License: (a) (i) This work is licensed under a Creative Commons Attribution 4.0 International License. Read Full License 


\section{Abstract}

Migrants and refugees are considered vulnerable to mental health problems and substance use disorders; and may be particularly affected by service disruptions associated with the COVID-19 pandemic The International Society of Addiction Medicine (ISAM) ran a multi-phased global survey among clinicians and health professional that are actively working in the field of addiction medicine to investigate the impact of the COVID-19 pandemic on substance use and related services. In March 2020, the first month after the announcement of the pandemic by the World Health Organization, 177 informants from 77 countries took part in the global survey, and only $12.9 \%$ of them reported their countries' substance use treatment and harm reduction services for the migrants and refugees with substance use disorders continued as usual. In May 2020, 11.7\% of respondents of the second phase reported that the services for refugees and migrants improved in comparison to March 2020; $11.7 \%$ reported that these services in their country discontinued. Results suggest that refugee and migrants access to treatment and harm reduction services has been reduced as a result of COVID-19. It can be concluded that it is crucial to improve the visibility of migrants' needs and exploit appropriate interventions for those with substance use disorders.

\section{Background}

As defined by the United Nations (UN), an international migrant is someone who changes his or her country of usual residence, irrespective of the reason for migration or legal status (1). Refugees are also persons who are residing outside of their country of origin for reasons of feared persecution, conflict, generalized violence, or other circumstances that have seriously disturbed public order and, as a result, require international protection (2). There are around 272 million migrants globally, majorly labour migrants (3), and amongst these groups, nearly 26 million are refugees, and half of them are under the age of 18 (4). Migrants and refugees are a heterogeneous group. As a result of selfselection (e.g., health, level of education, wealth, ambition, etc.) and the exclusion of unhealthy individuals at immigration pre-screening level, international migrant populations may have a better health condition than the native population (5-7). Overall they do not necessarily exhibit a significantly higher prevalence of physical and psychological morbidities, especially substance use disorder $(8,9)$. However, refugees often face a number of resettlement stressors and acculturations that may be compounded by the previous violence of trauma exposure, which cumulatively might increase the risk for substance use disorder. More importantly, being kept in detention centers (10), the stress of relocation (11), lesser access to the necessary services (12), unemployment, difficult living conditions, problems with learning a new language (13), isolation, separation anxiety and division of the family, living in violent or poor neighborhoods which drugs are more likely to be available, exposure to different types of trauma (11), discrimination and exclusion (14-16) transformation of social roles, weakened enforcement of substance control policies (17) and the effect of cultural shaping of symptoms and illness behaviors are all risk factors that may make migrants and refugees more vulnerable to mental health problems and substance use disorder $(18,19)$.

Migrants may be particularly affected by income loss, isolation, lockdown, insecurity, social exclusion and lack of access to culturally and linguistically accessible services, compounding further the vulnerabilities that migrants face on a daily basis $(20,21)$.

Published evidence demonstrates that people with substance use disorder (PWSUD) are at increased risk of COVID-19 and its associated complications (22). On the other hand substance use disorder (SUD) is considered to get more complicated during the COVID-19 pandemic (25). Migrant and refugee PWSUD are doubly at risk of exclusion and stigmatization.

At the time of the outbreak of COVID-19 (and before the announcement of the pandemic by the World Health Organization (WHO)), the International Society of Addiction Medicine (ISAM) published a set of guidelines on supporting clinicians and policymakers to deliver an effective service for people with substance use disorders (PWSUD) (24). One of the important recommendations in this manuscript was the prioritization of specific populations at risk, including refugees and migrants in the context of competing health priorities. Additionally, a multi-phase global survey was conducted by the ISAM COVID-19 team in order to better understand the impact the acute stages of COVID19 had on treatment and harm reduction services to PWSUD populations and their responses. The details of the methodology have been published as a study protocol (22). The participants consisted of nationally-representative informants from different countries who were experts in the field of addiction medicine and active in substance use treatment at different levels of policy and/or service during the pandemic. They were surveyed about the SUD treatment and harm reduction services for migrants and refugees collectively, during the COVID-19 pandemic in their country or region and the way it has been affected by the situation.

In total, 177 individuals from 77 countries participated in this ISAM survey. The survey results showed that in the first month of the pandemic announcement, substance use disorder treatment and harm reduction services were available and accessible in $59 \%$ of the participating countries. $88 \%$ of total respondents continued providing necessary medical and psychiatric care for PWSUD (20).

The availability and provision of SUD treatment and harm reduction services for refugees and migrants during COVID-19 pandemic in May 2020 is illustrated through a world map in Figure 1. The results of the first phase of the survey in March 2020 (the first month after the 
announcement of the pandemic by WHO), after excluding the unrelated responses which indicated lack of information or unwillingness to answer the question by the respondent, showed that only $12.9 \%(n=17)$ of respondents believed their countries' services for the migrants and refugees with substance use disorders continued as usual. In this time, services for this population were reported to be unavailable by $29.7 \%(n=39)$ of the respondents. $57.2 \%(n=75)$ of the respondents claimed that services are available for migrants and refugees in their countries but with limited access (Figure 2). Survey responses on harm reduction and treatment services for general population PWSUD showed that $43 \%(n=74)$ of the respondents reported that service provision is limited and not enough, whereas $26 \%(n=45)$ believed that both harm reduction and treatment services for general population PWSUD are adequately available and with enough coverage. $23 \%$ $(n=40)$ and $7 \%(n=13)$ of the respondents reported enough coverage and accessibility but only for treatment services, and only harm reduction services, respectively.

In May 2020, two months after the announcement of a pandemic situation, only $11.7 \%(n=8)$ of informant respondents of the second phase of the survey reported that the services for refugees and migrants with SUD got better in comparison to March 2020, whereas $11.7 \%$ $(n=8)$ reported that these services in their country discontinued completely and $41.1 \%(n=28)$ believed they got worse in comparison to their first phase reports. $41.1 \%(n=24)$ of the respondents reported that services for migrants and refugees continued as usual despite the pandemic (Figures 3,4 ). Respondents reported that service improvement rates were considerably lower for refugee and migrant subgroups relative to the whole population with SUD.

Although these findings were not derived from epidemiological data, they revealed that marginalized people, especially migrants and refugees, are among the groups that got most negative impact during COVID-19 even compared to the other vulnerable groups such as women, pregnant women, and children, this situation could be possibly due to higher risk of being neglected or even being sacrificed by policymakers and service providers.

Posselt et al (2017) (15) believed barriers of the services to refugees are related to four broad areas: 1- organizational and structural, 2access and engagement, 3- treatment and service delivery and 4- training and resources (15) which all mutually influence each other (26). It is therefore important to improve the visibility of the needs of migrants and appropriate interventions to those with substance use disorders by:

1. Raising awareness

2. Considering this group in service providers' business continuity measures,

3. Allocating enough resources to support this population during crisis situations

4. Enhancing collaboration and integration among various services

5. Advocacy for access to substance use disorders service

6. Reducing shame and stigma, and

7. Integrating substance use disorders treatment into other health services.

\section{Abbreviations}

COVID-19

Coronavirus Disease 2019

WHO

World Health Organization

UN

United Nations

ISAM

International Society of Addiction Medicine

SUD

Substance Use Disorder

PWSUD

People with Substance Use Disorders

\section{Declarations}

Ethics approval: The proposal was submitted to the University of Social Welfare and Rehabilitation Sciences, Tehran, Iran, and received the full approval of the ethics committee (IR.USWR.REC.1399.061). 
Consent for publication: Not applicable

Availability of data and materials: The datasets used and/or analyzed during the current study are available from the corresponding author on reasonable request.

Competing interests: The authors declare that they have no competing interests.

Funding: This study received no external funding resources.

Acknowledgements: The authors would like to thank the 177 members of the ISAM-PPIG consortium who responded to the survey.

Authors' contribution: All authors read and approved the final manuscript.

Authors' information: Anja Busse is a staff member of UNODC. The authors alone are responsible for the views expressed in this article and they do not necessarily represent the decisions or policies of the UNODC.

\section{References}

1. The UN department for economic and social affairs. https://refugeesmigrants.un.org/definitions. Accessed 20/11/2020

2. The UN high commissioner for refugees. https://refugeesmigrants.un.org/definitions. Accessed 20/11/2020

3. World Migration Report, UN, 2020. https://www.un.org/sites/un2.un.org/files/wmr_2020.pdf\&sa=D\&ust=1605990931185000\&usg=AOvVaw0n6VnMdw2mqnA2Ixh2bFwl

4. The UN refugee agency [https://www.unhcr.org/figures-at-a-glance.html. Accessed 30/10/2020

5. Kennedy S, Kidd MP, McDonald JT, Biddle N. The healthy immigrant effect: patterns and evidence from four countries. Journal of International Migration and Integration. 2015;16(2):317-32.

6. Singh GK, Hiatt RA. Trends and disparities in socioeconomic and behavioural characteristics, life expectancy, and cause-specific mortality of native-born and foreign-born populations in the United States, 1979-2003. International journal of epidemiology. 2006;35(4):903-19.

7. Agic B. Mental health of Canada's immigrants: Toronto, ON: Centre for Addiction and Mental Health. Retrieved from http ...; 2015.

8. Harris S, Dykxhoorn J, Hollander A-C, Dalman C, Kirkbride JB. Substance use disorders in refugee and migrant groups in Sweden: A nationwide cohort study of 1.2 million people. PLoS medicine. 2019;16(11):e1002944.

9. Luitel NP, Jordans M, Murphy A, Roberts B, McCambridge J. Prevalence and patterns of hazardous and harmful alcohol consumption assessed using the AUDIT among Bhutanese refugees in Nepal. Alcohol and alcoholism. 2013;48(3):349-55.

10. Silove D, Austin P, Steel Z. No refuge from terror: the impact of detention on the mental health of trauma-affected refugees seeking asylum in Australia. Transcultural psychiatry. 2007;44(3):359-93.

11. Danso R. From 'there'to 'here': An investigation of the initial settlement experiences of Ethiopian and Somali refugees in Toronto. GeoJournal. 2002;56(1):3-14.

12. Mukumbang FC, Ambe AN, Adebiyi BO. Unspoken inequality: how COVID-19 has exacerbated existing vulnerabilities of asylum-seekers, refugees, and undocumented migrants in South Africa. International Journal for Equity in Health. 2020;19(1):141.

13. addiction center [https://www.addictioncenter.com/addiction/refugees-immigrants/ Accessed 20 oct 2020

14. Colic-Peisker V, Tilbury F. Integration into the Australian labour market: the experience of three "visibly different" groups of recently arrived refugees 1. International migration. 2007;45(1):59-85.

15. Fozdar F, Hartley L. Civic and ethno belonging among recent refugees to Australia. Journal of refugee studies. 2014;27(1):126-44.

16. Fosados R, McClain A, Ritt-Olson A, Sussman S, Soto D, Baezconde-Garbanati L, et al. The influence of acculturation on drug and alcohol use in a sample of adolescents. Addictive behaviors. 2007;32(12):2990-3004.

17. Weissbecker I, Hanna F, El Shazly M, Gao J, Ventevogel P. Integrative mental health and psychosocial support interventions for refugees in humanitarian crisis settings. An uncertain safety: Springer; 2019. p. 117-53.

18. Posselt M, McDonald K, Procter N, de Crespigny C, Galletly C. Improving the provision of services to young people from refugee backgrounds with comorbid mental health and substance use problems: addressing the barriers. BMC public health. 2017;17(1):280.

19. Sundquist K, Frank G. Urbanization and hospital admission rates for alcohol and drug abuse: a follow-up study of 4.5 million women and men in Sweden. Addiction. 2004;99(10):1298-305. 
20. Kluge, H. H. P., Jakab, Z., Bartovic, J., D'Anna, V., \& Severoni, S. (2020). Refugee and migrant health in the COVID-19 response. The Lancet, 395(10232), 1237-1239.

21. Wang, Q.Q., Kaelber, D.C., Xu, R. et al. COVID-19 risk and outcomes in patients with substance use disorders: analyses from electronic health records in the United States. Mol Psychiatry (2020). https://doi.org/10.1038/s41380-020-00880-7

22. Baldacchino A, Radfar SR, De Jong C, Rafei P, Yunesian M, Gerra G, et al. COVID-19 and Substance Use Disorder: Study Protocol for the International Society of Addiction Medicine Practice and Policy Interest Group Global Survey. Basic and clinical neuroscience. 2020;11(2):155.

23. Volkow ND. Collision of the COVID-19 and addiction epidemics. American College of Physicians; 2020.

24. Farhoudian A, Baldacchino A, Clark N, et al. COVID-19 and Substance Use Disorders: Recommendations to a Comprehensive Healthcare Response. An International Society of Addiction Medicine Practice and Policy Interest Group Position Paper. Basic Clin Neurosci. 2020;11(2):133-150. doi:10.32598/bcn.11.covid19.1

25. Radfar SR, De Jong CA, Farhoudian A, Ebrahimi M, Rafei P, Vahidi M, et al. Reorganization of Substance Use Treatment and Harm Reduction Services during the COVID-19 Pandemic: A Global Survey. medRxiv. 2020.

26. Hobfoll SE. Conservation of resources and disaster in cultural context: The caravans and passageways for resources. Psychiatry: Interpersonal \& Biological Processes. 2012;75(3):227-32.

\section{Figures}

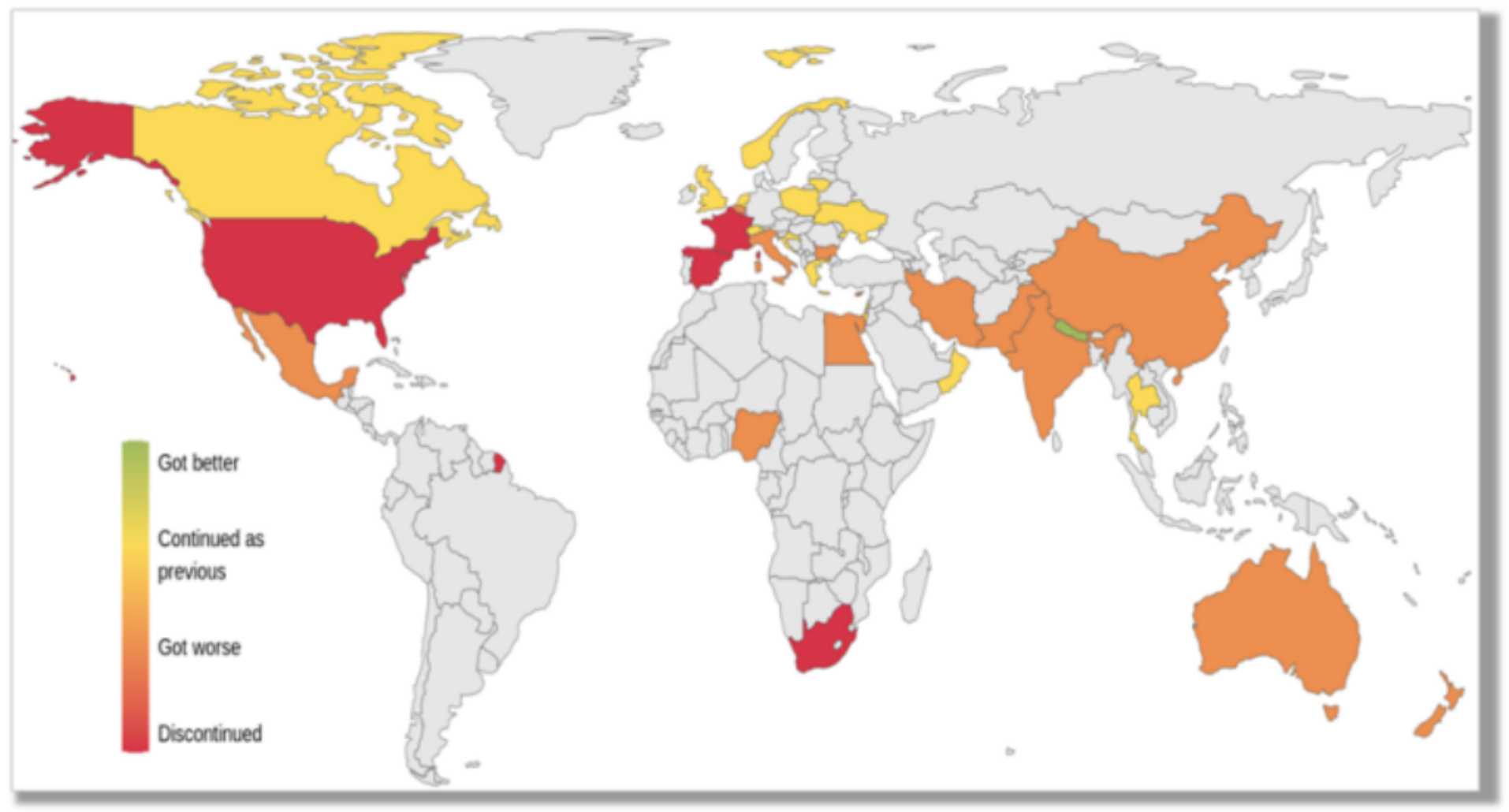

\section{Figure 1}

Status of the 77 participating countries in the first phase of the survey depicting changes in availability and provision of substance use treatment and harm reduction services for migrants and refugees with substance use disorders in March 2020. Note: The designations employed and the presentation of the material on this map do not imply the expression of any opinion whatsoever on the part of Research Square concerning the legal status of any country, territory, city or area or of its authorities, or concerning the delimitation of its frontiers or boundaries. This map has been provided by the authors. 


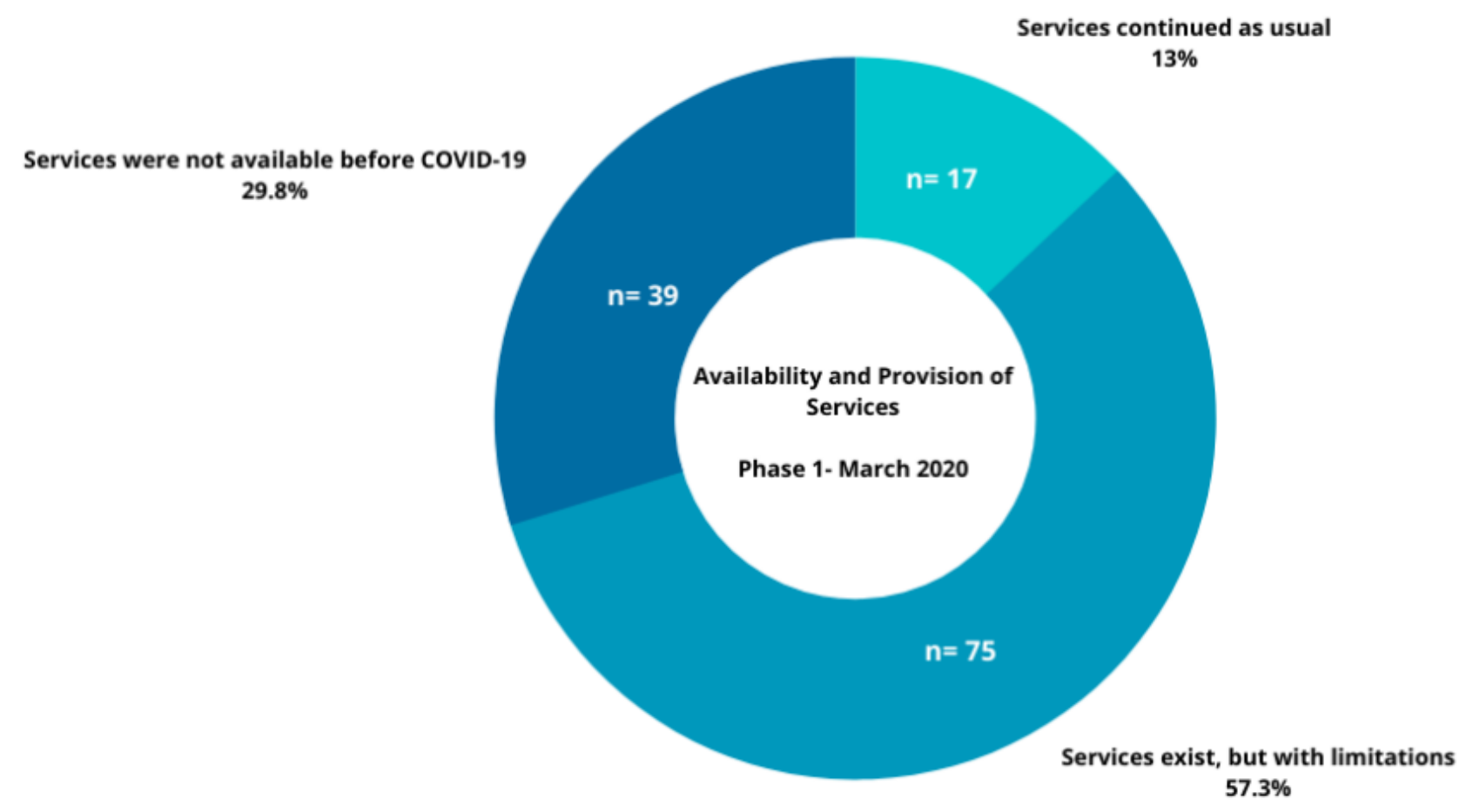

Figure 2

Availability and provision of treatment and harm reduction services for migrants and refugees with substance use disorders in March 2020 reported by 177 respondents from 77 countries.

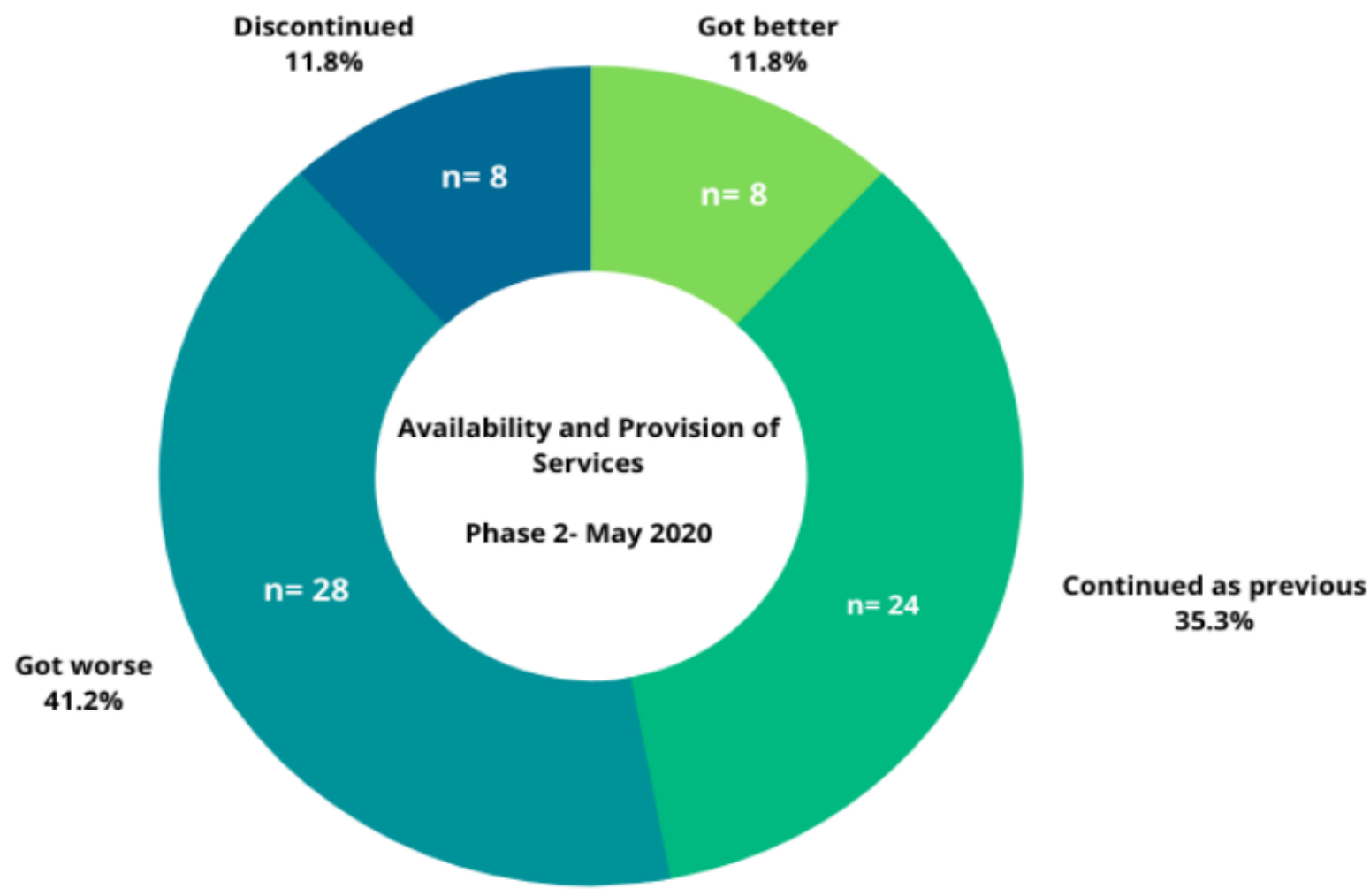

\section{Figure 3}

Changes in availability and provision of substance use treatment and harm reduction services for migrants and refugees with substance use disorders in May 2020 reported by 105 respondents from 52 countries. 


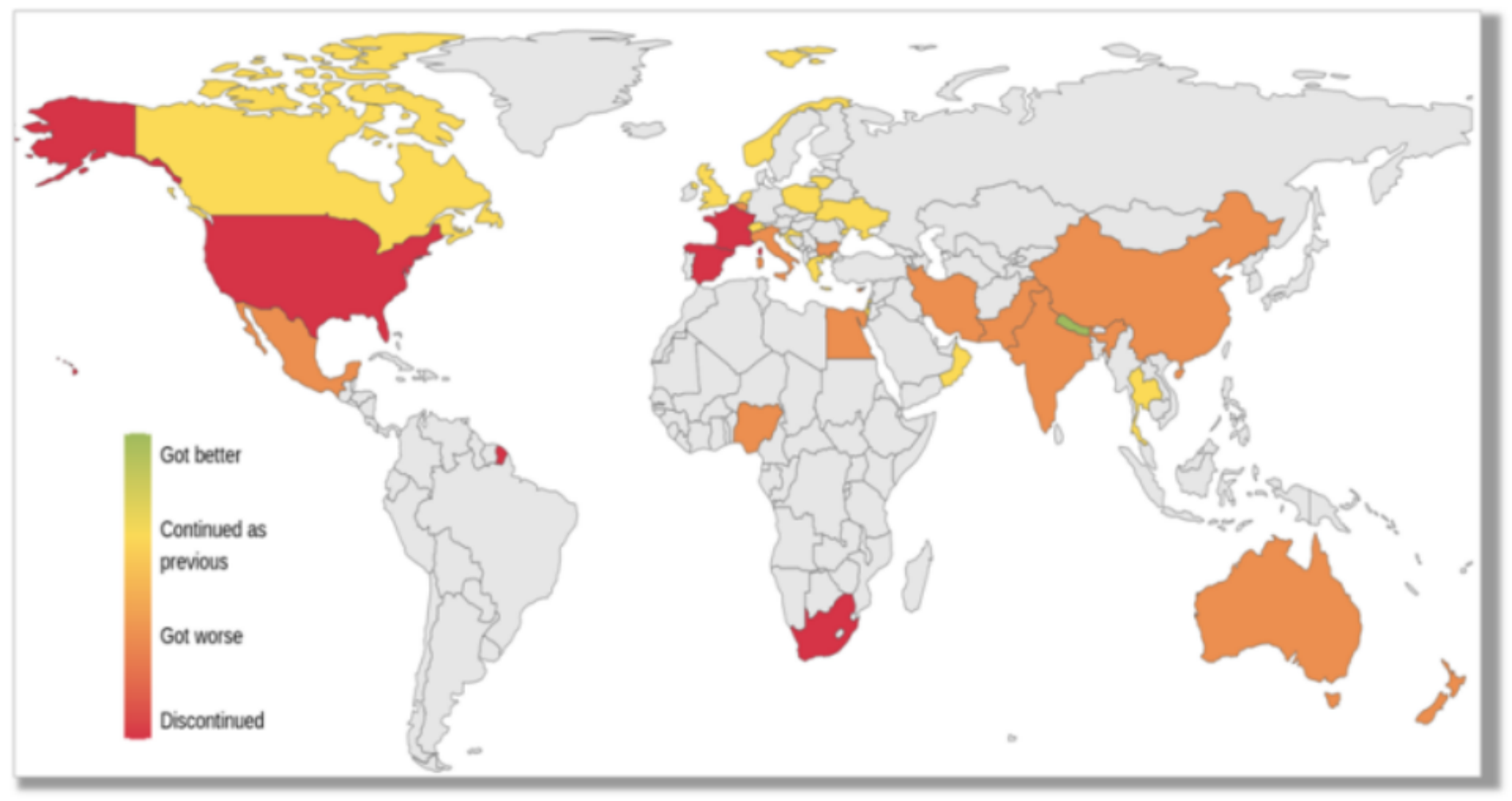

\section{Figure 4}

Status of the 52 participating countries in the second phase of the survey depicting changes in availability and provision of substance use treatment and harm reduction services for migrants and refugees with substance use disorders in May 2020. Note: The designations employed and the presentation of the material on this map do not imply the expression of any opinion whatsoever on the part of Research Square concerning the legal status of any country, territory, city or area or of its authorities, or concerning the delimitation of its frontiers or boundaries. This map has been provided by the authors. 